\title{
Violência contra a mulher na vida: estudo entre usuárias da Atenção Primária
}

\author{
Violence against women in life: study among Primary Care users
}

Ione Barbosa dos Santos (https://orcid.org/0000-0002-2513-7623) ${ }^{1}$

Franciéle Marabotti Costa Leite (https://orcid.org/0000-0002-6171-6972) ${ }^{2}$

Maria Helena Costa Amorim (https://orcid.org/0000-0002-4252-7092) ${ }^{2}$

Paulete Maria Ambrósio Maciel (https://orcid.org/0000-0002-2141-7732) ${ }^{2}$

Denise Petrucci Gigante (https://orcid.org/0000-0001-7309-5838) ${ }^{3}$
${ }^{1}$ Secretaria Municipal de Saúde, Prefeitura Municipal de Vitória. Av. Mal.

Mascarenhas de Moraes

1185, Forte São João. 28017010 Vitória ES Brasil.

ilubarsantos@hotmail.com

${ }^{2}$ Programa de Pós-

Graduação em Enfermagem, Departamento de

Enfermagem, Universidade Federal do Espírito Santo.

Vitória ES Brasil.

${ }^{3}$ Programa de Pós-

Graduação em

Epidemiologia,

Departamento de Nutrição,

Universidade Federal de

Pelotas. Pelotas RS Brasil.
Abstract This article aims to estimate the prevalence and factors associated with intimate partner violence among primary care users. Crosssectional study with women aged 20 to 59 years. Physical, sexual and psychological violence was screened by the World Health Organization instrument. Poisson regression was used for crude and adjusted analysis. Nine hundred ninety-one women participated in the study. The prevalence of violence throughout the lifespan was: psychological 57.6\% (95\%CI 54.6-60.7); physical 39.3\% (95\%CI 36.2-42.3) and sexual $18.0 \%$ (95\%CI 15.7-20.5). Women with up to eight years of schooling, divorced or separated, whose mothers suffered intimate partner violence, who reported drug use and experienced sexual violence in childhood showed a higher prevalence of the three types of violence. Religion was associated with psychological and sexual violence and the use of cigarettes to physical and psychological violence. Participants with lower household income had a higher prevalence of physical violence. A high prevalence of intimate partner violence was identified among users. Worse socioeconomic conditions, risk behaviors and a history of assault are associated with greater occurrence of this problem.

Key words Violence, Socioeconomic factors, $\mathrm{Ma}$ rital abuse, Intimate partner violence, Domestic violence
Resumo $O$ objetivo deste artigo é estimar a prevalência e os fatores associados à violência praticada por parceiro intimo, ao longo da vida, entre as usuárias da Atenção Primária. Estudo transversal com mulheres de 20 a 59 anos. O rastreio das violências foi feito pelo instrumento da Organização Mundial da Saúde. Regressão de Poisson foi utilizada para análise bruta e ajustada. Participaram 991 mulheres. As prevalências de violência, ao longo da vida, foram: psicológica 57,6\% (IC95\%: 54,6-60,7); física 39,3\% (IC95\% 36,2-42,3) e sexual 18,0\% (IC95\% 15,7-20,5). Mulheres com até oito anos de estudo, divorciadas ou separadas, cujas mães sofreram violência por parceiro intimo, que relataram uso de drogas e vivenciaram a violência sexual na infância apresentaram maiores prevalências das três violências. A religião esteve associada às violências psicológica e sexual e o uso de cigarro às violências física e psicológica. Participantes de menor renda familiar tiveram maiores prevalências de violência física. Elevada prevalência de violência cometida pelo parceiro íntimo ao longo da vida entre as usuárias. Piores condições socioeconômicas, comportamentos de risco e histórico de agressão estão associados à maior ocorrência deste agravo.

Palavras-chave Violência, Fatores socioeconômicos, Maus-tratos conjugais, Violência por parceiro intimo, Violência doméstica 


\section{Introdução}

A violência contra a mulher, constitui um fenômeno complexo, baseado nas relações de gênero, que desencadeia danos físicos, psicológicos e/ou sofrimento para a mulher ${ }^{1}$. Esse agravo é um problema mundial, e, está baseado nas relações desiguais de poder, ou seja, constitui um tipo de violência motivada pela condição desigual de sexo, a qual começa no universo familiar, e as relações de gênero se estabelecem de forma hierárquica ${ }^{2}$.

Segundo a Organização Mundial da Saúde, em 2013, 30,0\% das mulheres no mundo já haviam sido vítimas de violência praticada pelo parceiro íntimo ${ }^{3}$. No Brasil, estudo de base populacional realizado com amostra representativa de 15 anos ou mais mostrou que $43 \%$ das brasileiras declararam ter sofrido violência praticada por um homem na vida; um terço admitiu ter sofrido alguma forma de violência física, 13\% sexual e $27 \%$ psicológica. Maridos, ex-maridos, namorados e ex-namorados foram os principais agressores, variando de $88 \%$ dos autores de tapas e empurrões a $79 \%$ dos perpetradores de relações sexuais forçadas ${ }^{4}$.

Esse fenômeno social de elevada prevalência, afeta sobremaneira a saúde e o modo de viver das mulheres ${ }^{5}$. Os danos causados por essa violência podem durar uma vida inteira e atingir gerações, com efeitos graves na saúde, educação, trabalho, crime e bem-estar econômico de indivíduos, famílias, comunidades e sociedades ${ }^{6}$, constituindo assim, uma grande preocupação para a população brasileira e para o setor da saúde, uma vez que pode acarretar mortes, traumas físicos e emocionais $^{7}$. Dessa maneira, é de fundamental importância o conhecimento e postura adequada dos profissionais no atendimento às vítimas, identificando e notificando os casos, uma vez que o serviço de saúde tem o dever de acolher e apoiar, e não ser mais um obstáculo para as vítimas.

Entretanto, a literatura aponta que o sofrimento da mulher em situação de violência ainda não é visto como elemento que mereça intervenção dos profissionais da área da saúde, a não ser que haja alguma base anatomopatológica objetiva para justificá-lo. Neste último caso, a interpretação de doença pode ser acolhida, e a violência perde sentido e importância, tendendo a ser desconsiderada ${ }^{7}$. As práticas das equipes da atenção básica frente às situações de violência doméstica ainda são desafiadoras e constituem um dilema para a construção da atenção integral. O despreparo dos profissionais em lidar com situações de violência se soma à própria concepção dos profissionais sobre o fenômeno e suas causas, as ações desenvolvidas (ou não) e a relação que se estabelece com a família e demais atores da rede intersetorial".

Diante do exposto, considerando a violência um agravo de saúde e a não existência de estudos que abordem a ocorrência desse fenômeno ao longo da vida da mulher no município de Vitória, o objetivo dessa pesquisa foi estimar a prevalência e os fatores associados à violência praticada por parceiro íntimo ao longo da vida entre as usuárias da Atenção Primária.

\section{Método}

Trata-se de um estudo epidemiológico, transversal, realizado com dados de uma pesquisa maior sobre a violência contra a mulher no município de Vitória, Espírito Santo, feita no período de março a setembro de 2014, em todas as unidades de saúde (US) do referido município, que apresentavam Estratégia de Saúde da Família (ESF) e/ ou Programa de Agentes Comunitários de saúde (PACS), totalizando 26 unidades. O município de Vitória, capital do ES tem 327.801 mil habitantes ${ }^{10}$, sendo o Índice de Desenvolvimento Humano (IDH) do município de $0,845^{11}$.

O cálculo do tamanho da amostra considerou aceitável uma margem de erro de cinco pontos percentuais e nível de $95 \%$ de confiança. Para estudar a associação com os fatores de risco, considerou-se um nível de $95 \%$ de confiança, poder de $80 \%$ e razão exposto/não exposto de 1:1. Foram acrescidos $10 \%$ para possíveis perdas e 30\% para análises ajustadas, sendo necessário um total de 998 mulheres. A técnica de amostragem utilizada foi amostragem proporcional por unidade de saúde.

As mulheres foram abordadas na US, convidadas a participar da pesquisa e, após o aceite e assinatura do Termo de Consentimento Livre e Esclarecido (TCLE), eram entrevistadas, de forma individualizada, com apenas a entrevistada e a entrevistadora, devidamente treinada, em um espaço reservado da US. Foram incluídas no estudo usuárias que possuíam parceiro íntimo (VPI) no momento da entrevista ou nos últimos 12 meses. Foi definido como parceiro íntimo o companheiro ou ex-companheiro, independentemente da união formal, e namorados atuais, desde que mantendo relações sexuais. Como critério de exclusão adotou-se: mulheres com déficit intelectual ou sensorial incapazes de 
se comunicarem. Para essa pesquisa utilizou-se o banco contendo a caracterização socioeconômica: idade (categorizada por décadas); cor da pele autorreferida (branca, preta e parda), sendo excluídas as indígenas ou de origem asiática por constituírem um grupo muito pouco representado não sendo possível qualquer inferência dos resultados; escolaridade (até oito anos; nove anos ou mais); renda familiar no momento da entrevista $(>=1.500 ; 1.501-2.924 ;<2.925)$; situação conjugal (casada; solteira; divorciada ou separada; e em união consensual); religião (católica ou evangélica: sim/não). Para identificar a experiência familiar e vida de violência foi perguntado, respectivamente: "Sua mãe já sofreu alguma violência por parte do parceiro íntimo?' (sim/não) e "A senhora sofreu violência sexual na infância?" (sim/não). Quanto à caracterização comportamental foi questionado: dose de bebida alcoólica ingerida (menor ou igual a duas; mais de duas e até oito; ou mais de oito doses). Uma dose correspondeu, em média, a uma lata de cerveja ou chope de $350 \mathrm{ml}$, uma taça de vinho de $90 \mathrm{ml}$, uma dose de destilado de $30 \mathrm{ml}$, uma lata ou uma garrafa pequena de qualquer bebida "ice"; tabagismo (distribuído em duas categorias: fumantes: fumavam pelo menos um cigarro por dia; não fumantes: que incluiu ex-fumantes) e histórico de uso drogas (uso de drogas alguma vez na vida: sim/não).

O segundo instrumento aplicado foi a versão reduzida do questionário da Organização Mundial da Saúde (OMS), intitulado World Health Organization Violence Against Women (WHO VAW STUDY) ${ }^{12}$, validado para uso no Brasil, que contém 13 questões que determinam os desfechos em estudos: violências psicológica, física e sexual cometida pelo parceiro íntimo ao longo da vida. As violências foram consideradas presentes quando a mulher respondeu sim a um dos itens do instrumento. Vale destacar que ao final da entrevista todas as participantes recebiam um folder contendo os principais serviços de atendimento às mulheres em situação de violência e, se necessário, eram realizados encaminhamentos ao núcleo de atendimento às vítimas do município de Vitória.

Para análise dos dados utilizou-se o pacote estatístico Stata 13.0. Para a análise bivariada, que investigou a associação entre os desfechos e as exposições em estudo foi feita através do teste qui-quadrado ou exato de Fisher conforme pressupostos. A análise multivariada foi avaliada por meio da Regressão de Poisson, com variância robusta, bruta e ajustada para controle dos fatores de confusão segundo modelo hierárquico (Figura 1). Para a entrada no modelo não foi determinado um valor de $\mathrm{p}$, para não excluir possíveis fatores de confusão; já a permanência no modelo foi determinada pelo valor de $p<0,05$. O presente estudo foi aprovado pelo Comitê de Ética em Pesquisa da Universidade Federal do Espírito Santo.

\section{Resultados}

Foram entrevistadas 991 mulheres, ou seja, sete mulheres recusaram-se a participar do estudo. Dentre as participantes houve predomínio, ao longo da vida, da violência psicológica com prevalência de 57,6\% (IC95\%: 54,6-60,7). A violência física foi a segunda mais prevalente, com $39,3 \%$ (IC95\% 36,2-42,3). A violência com menor prevalência foi sexual, com 18,0\% (IC95\% 15,7-20,5) (dados não apresentados em Tabela).

Ao avaliar as prevalências das violências de acordo com as características em estudo (Tabela

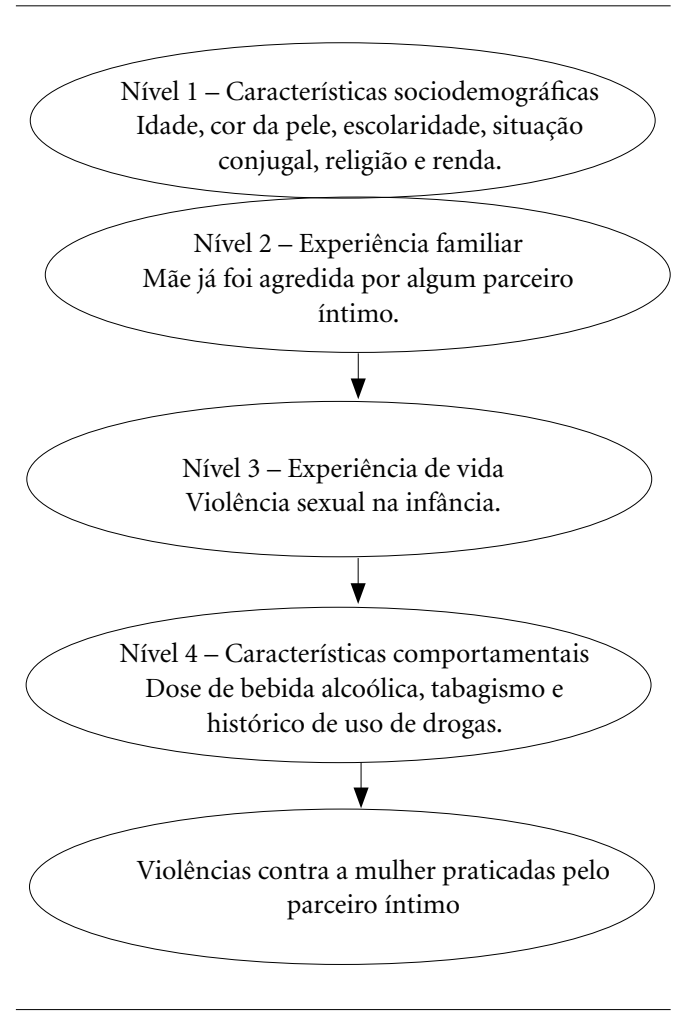

Figura 1. Modelo hierárquico das relações entre os fatores de risco para o desfecho das Violências Contra a Mulher praticadas pelo parceiro íntimo. 
Tabela 1. Prevalência das violências contra a mulher praticada pelo parceiro íntimo, ao longo da vida, de acordo com as características socioeconômicas, comportamentais, experiência familiar e de vida. Vitória, Espírito Santo, Brasil. Março a setembro, 2014.

\begin{tabular}{|c|c|c|c|c|c|c|}
\hline \multirow{2}{*}{$\begin{array}{c}\text { Características } \\
\text { sociodemográficas }\end{array}$} & \multicolumn{2}{|c|}{ Violência Psicológica } & \multicolumn{2}{|c|}{ Violência física } & \multicolumn{2}{|c|}{ Violência Sexual } \\
\hline & P (IC95\%) & p-valor & P (IC95\%) & p-valor & P (IC95\%) & p-valor \\
\hline Idade (anos) & & $0,529^{c}$ & & $0,612^{\mathrm{c}}$ & & $0,055^{\mathrm{c}}$ \\
\hline 20-29 & $54,4(48,6-60,1)$ & & $40,4(34,4-45,8)$ & & $15,4(11,7-20,1)$ & \\
\hline $30-39$ & $57,8(52,2-63,2)$ & & $36,6(31,4-42,1)$ & & $15,0(11,4-19,5)$ & \\
\hline $40-49$ & $58,7(52,1-64,9)$ & & $42,2(35,9-48,8)$ & & $22,7(17,6-28,6)$ & \\
\hline $50-59$ & $61,1(53,4-68,1)$ & & $38,8(31,9-46,3)$ & & $21,1(15,7-27,8)$ & \\
\hline Cor da pele ${ }^{a}$ & & $0,086^{\mathrm{c}}$ & & $0,038^{c}$ & & $0,910^{\mathrm{c}}$ \\
\hline Branca & $51,1(44,4-57,8)$ & & $32,5(26,6-39,1)$ & & $18,6(13,9-24,4)$ & \\
\hline Parda & $58,2(53,9,-62,4)$ & & $40,5(36,3-44,9)$ & & $17,3(14,2-20,9)$ & \\
\hline Preta & $61,09(54,7-67,1)$ & & $43,9(37,7-50,3)$ & & $18,0(13,6-23,4)$ & \\
\hline $\begin{array}{l}\text { Escolaridade (anos de } \\
\text { estudo) }\end{array}$ & & $<0,001^{\mathrm{c}}$ & & $<0,001^{\mathrm{c}}$ & & $<0,001^{\mathrm{c}}$ \\
\hline $0-8$ & $67,0(61,4-72,1)$ & & $51,1(45,5-56,8)$ & & $25,1(20,5-30,3)$ & \\
\hline 9 ou mais & $53,5(49,7-57,2)$ & & $34,0(30,5-37,6)$ & & $14,8(12,3-17,7)$ & \\
\hline Renda familiar (em tercil) & & $0,001^{d}$ & & $<0,001^{\mathrm{d}}$ & & $0,036^{d}$ \\
\hline$>=1500$ & $65,6(60,4-70,4)$ & & $48,7(43,4-54,0)$ & & $13,9(10,6-18,1)$ & \\
\hline $1501-2924$ & $55,0(49,5-60,4)$ & & $42,1(36,8-47,6)$ & & $21,6(17,5-26,6)$ & \\
\hline$<2925$ & $51,8(46,4-57,2)$ & & $26,7(22,1-31,7)$ & & $18,4(14,6-22,8)$ & \\
\hline Situação conjugal & & $<0,001^{\mathrm{c}}$ & & $<0,001^{\mathrm{c}}$ & & $0,027^{\mathrm{c}}$ \\
\hline Casada & $48,2(43,5-52,9)$ & & $27,2(23,2-31,5)$ & & $14,6(11,6-18,2)$ & \\
\hline União consensual & $63,1(57,4-68,4)$ & & $47,1(41,4-52,8)$ & & $19,3(15,2-24,2)$ & \\
\hline Solteira & $66,0(59,7-71,7)$ & & $49,2(42,8-55,5)$ & & $21,0(16,3-26,7)$ & \\
\hline Divorciada/separada & $85,0(61,6-95,2)$ & & $70,0(46,7-86,2)$ & & $35,0(17,3-58,1)$ & \\
\hline Católica & & $0,273^{\mathrm{c}}$ & & $0,846^{\mathrm{c}}$ & & $0,585^{\mathrm{c}}$ \\
\hline Sim & $55,6(50,1-60,3)$ & & $38,9(34,3-43,7)$ & & $17,2(13,9-21,1)$ & \\
\hline Não & $59,1(55,0-63,0)$ & & $39,5(35,6-43,6)$ & & $18,5(15,5-21,9)$ & \\
\hline Evangélica & & $0,110^{\mathrm{c}}$ & & $0,031^{\mathrm{c}}$ & & $0,034^{\mathrm{c}}$ \\
\hline Sim & $60,2(55,8-64,5)$ & & $42,7(38,3-47,2)$ & & $20,6(17,2-24,5)$ & \\
\hline Não & $55,2(50,1-64,5)$ & & $36,0(32,0-40,3)$ & & $15,5(12,6-18,9)$ & \\
\hline \multicolumn{7}{|l|}{$\begin{array}{l}\text { Experiência familiar e de } \\
\text { vida }\end{array}$} \\
\hline $\begin{array}{l}\text { Mãe já apanhou de algum } \\
\text { parceiro }^{\text {b }}\end{array}$ & & $<0,001^{\mathrm{c}}$ & & $<0,001^{\mathrm{c}}$ & & $0,003^{\mathrm{c}}$ \\
\hline Sim & $66,8(61,4-71,8)$ & & $48,2(42,7-53,8)$ & & $22,7(18,4-27,7)$ & \\
\hline Não & $51,6(47,6-55,7)$ & & $32,8(29,1-36,7)$ & & $14,7(12,0-17,8)$ & \\
\hline Violência sexual na infância & & $0,001^{\mathrm{c}}$ & & $0,100^{\mathrm{c}}$ & & $<0,001^{\mathrm{c}}$ \\
\hline Sim & $71,9(63,2-79,2)$ & & $57,0(48,9-65,6)$ & & $37,2(29,0-46,2)$ & \\
\hline Não & $55,6(52,3-58,9)$ & & $36,8(33,6-40,0)$ & & $15,3(13,0-17,8)$ & \\
\hline \multicolumn{7}{|l|}{$\begin{array}{l}\text { Características } \\
\text { comportamentais }\end{array}$} \\
\hline Dose de bebida alcoólica & & $0,016^{\mathrm{c}}$ & & $0,001^{\mathrm{c}}$ & & $0,056^{\mathrm{c}}$ \\
\hline Nenhuma & $57,3(53,5-61,1)$ & & $37,2(33,6-41,0)$ & & $20,3(17,3-23,5)$ & \\
\hline$<=2$ & $48,2(40,1-56,5)$ & & $35,5(28,0-43,7)$ & & $11,4(7,1-17,8)$ & \\
\hline $2,1-8,0$ & $62,9(54,7-70,5)$ & & $42,0(34,1-50,2)$ & & $14,7(9,8-21,5)$ & \\
\hline$>8,0$ & $69,2(56,9-79,3)$ & & $61,5(49,1-72,6)$ & & $16,9(9,6-28,2)$ & \\
\hline Fumante & & $<0,001^{\mathrm{c}}$ & & $<0,001^{\mathrm{c}}$ & & $0,050^{c}$ \\
\hline Sim & $77,1(68,2-84,0)$ & & $65,1(55,7-73,5)$ & & $24,8(17,5-33,8)$ & \\
\hline Não & $55,2(51,9-58,5)$ & & $36,1(32,9-39,3)$ & & $17,1(14,8-19,7)$ & \\
\hline Histórico de uso drogas & & $<0,001^{\mathrm{c}}$ & & $<0,001^{\mathrm{c}}$ & & $<0,001^{\mathrm{c}}$ \\
\hline Sim & $85,8(77,8-91,3)$ & & $73,6(64,3-81,1)$ & & $34,9(26,4-44,5)$ & \\
\hline Não & $54,2(50,9-57,5)$ & & $35,1(32,0-38,3)$ & & $15,9(13,7-18,5)$ & \\
\hline
\end{tabular}


1), observaram-se maiores prevalências de abuso psicológico, físico e sexual cometido pelo parceiro, na vida, entre mulheres com até oito anos de estudos, pertencentes ao grupo de menor renda familiar, divorciadas/separadas, cuja mãe sofreu violência por parceiro íntimo, fumantes e com histórico de uso de drogas. Mulheres evangélicas apresentaram maiores frequências de violência física e sexual. Participantes da pesquisa com história de violência sexual na infância tiveram maior ocorrência de abuso psicológico e sexual cometido pelo parceiro ao longo da vida. Além disso, mulheres que ao beber ingerem mais de oito doses de bebida alcoólica estão entre o grupo com mais prevalência das violências psicológica $\mathrm{e}$ física $(\mathrm{p}<0,05)$.

A Tabela 2 apresenta a análise bruta e ajustada dos efeitos das variáveis socioeconômica, comportamentais e experiência familiar e de vida sobre a violência psicológica praticada pelo parceiro ao longo da vida. Nota-se, após ajuste, que a violência psicológica manteve-se associada à escolaridade, situação conjugal, religião evangélica, histórico materno de VPI, violência sexual na infância, tabagismo e uso de drogas (p $<0,05)$. Mulheres com até oito anos de estudos, cuja mãe sofreu algum tipo de violência por parceiro íntimo, que foram abusadas sexualmente na infância ou são fumantes apresentam cerca de $20 \%$ mais frequência de abuso psicológico pelo parceiro, ao longo da vida, quando compradas àquelas com maior escolaridade, sem histórico materno e pessoal de violência e não tabagistas. Do mesmo modo, a prevalência desse agravo foi $70 \%$ maior entre as mulheres separadas ou divorciadas quando comparadas às mulheres casadas (RP: 1,70; IC95\%: 1,37-2,10). Mulheres que já usaram drogas têm 1,37 vezes mais ocorrência de violência psicológica comparadas àquelas que nunca utilizaram (RP: 1,37; IC95\%: 1,21-1,55). Observa-se ainda que ter referido ser da religião evangélica aumentou em $12 \%$ a prevalência da violência psicológica (RP: 1,12; IC95\%: 1,01$1,24)$.

Após ajustes para os fatores de confusão, verifica-se que a violência física perpetrada pelo parceiro, na vida, foi quase 2,27 vezes mais prevalente em mulheres divorciadas e separadas, quando compradas àquelas casadas. Quanto à renda familiar e à escolaridade, observa-se um aumento desse tipo de abuso em $41 \%$ entre as mulheres do grupo de menor renda (menor ou igual a $\mathrm{R} \$ 1.500,00 / \mathrm{mês})$, quando comparadas ao de maior (maior que $\mathrm{R} \$ 2.925,00 / \mathrm{mês}$ ), e de $33 \%$ entre aquelas com menor escolaridade (até oito anos de estudo). Mulheres que fumam e com histórico de uso de drogas estão entre os grupos com maior prevalência de violência física, respectivamente: $34 \%$ e $64 \%$ quando comparadas àquelas que não relataram tais comportamentos. Quanto à experiência familiar e de vida, constata-se que, entre aquelas cuja mãe já apanhou do parceiro, há um aumento em $27 \%$. Já entre as que têm história de violência sexual na infância, a prevalência de violência física é $45 \%$ maior comparado a quem não sofreu esse abuso na infância (Tabela 3).

Na Tabela 4, observa-se a análise bruta e ajustada para violência sexual por parceiro íntimo ao longo da vida. Constata-se, após o ajuste, entre as divorciadas ou separadas, uma prevalência 2,18 vezes maior dessa violência, se comparadas às mulheres casadas. Aquelas com até oito anos de escolaridade têm $63 \%$ mais ocorrência de abuso sexual em comparação às de escolaridade maior. A violência sexual foi ainda duas vezes mais prevalente em mulheres que sofreram abuso sexual na infância e já utilizaram drogas, quando comparadas com aquelas que negaram esses fatos. Outro ponto a destacar é o aumento em 33\% do abuso sexual por parceiro íntimo, na vida, em mulheres da religião evangélica, comparadas às que não pertencem a esse grupo. Houve maior prevalência da violência sexual entre aquelas cuja mãe apanhou do parceiro íntimo (RP:1,37, IC95\%: 1,03-1,84).

\section{Discussão}

A presente pesquisa revela elevadas prevalências de violência por parceiro íntimo, ao longo da vida, entre usuárias de atenção primária à saúde no município de Vitória, sendo a violência psicológica a mais prevalente ( $\mathrm{P}=57,6 \%$ IC95\%: 54,6-60,7), confirmando os achados de um estudo transversal realizado em 2014, com mulheres na cidade de Recife, Pernambuco, onde o abuso psicológico também foi o mais frequente ${ }^{13}$. Para alguns autores, esse tipo de violência talvez seja mais facilmente compartilhado devido a fatores que podem envolver o medo de mais agressões ou vergonha de falar sobre abusos ocorridos na relação ${ }^{6}$. É importante destacar a relevância de se analisar a ocorrência da violência psicológica e as formas de preveni-la, visto ser considerada o ponto inicial que deflagra todas as outras formas de violência ${ }^{14}$.

O segundo tipo de abuso mais evidenciado foi a violência física ( $\mathrm{P}=39,3 \%$; IC95\% 36,2- 
Tabela 2 Análise bruta e ajustada dos efeitos das variáveis socioeconômica, comportamentais e experiência familiar e de vida sobre a violência psicológica perpetrada pelo parceiro íntimo ao longo da vida. Vitória, Espírito Santo, Brasil. Março a setembro, 2014.

\begin{tabular}{|c|c|c|c|c|}
\hline \multirow[b]{2}{*}{ Características sociodemográficas } & \multicolumn{2}{|c|}{ Análise bruta } & \multicolumn{2}{|c|}{ Análise ajustada } \\
\hline & $\begin{array}{l}\text { RP bruta } \\
\text { (IC95\%) }\end{array}$ & p-valor & $\begin{array}{c}\text { RP ajustada } \\
(\text { IC95\%) }\end{array}$ & p-valor \\
\hline Idade (anos) & & 0,532 & & 0,178 \\
\hline 20-29 & 1,0 & & 1,0 & \\
\hline $30-39$ & $1,06(0,92-1,23)$ & & $1,09(0,95-1,25)$ & \\
\hline $40-49$ & $1,08(0,93-1,26)$ & & $1,09(0,94-1,27)$ & \\
\hline $50-59$ & $1,12(0,96-1,31)$ & & $1,21(1,02-1,42)$ & \\
\hline Cor $^{\mathrm{a}}$ & & 0,103 & & 0,446 \\
\hline Branca & 1,0 & & 1,0 & \\
\hline Parda & $1,19(1,01-1,40)$ & & $1,11(0,94-1,31)$ & \\
\hline Preta & $1,14(0,98-1,32)$ & & $1,04(0,90-1,22)$ & \\
\hline Escolaridade (em anos de estudo) & & $<0,001$ & & $<0,001$ \\
\hline $0-8$ & $1,49(1,22-1,83)$ & & $1,23(1,10-1,36)$ & \\
\hline $9-11$ & 1,0 & & 1,0 & \\
\hline Renda familiar (em reais) & & $<0,001$ & & 0,191 \\
\hline$>=1500$ & $1,27(1,11-1,44)$ & & $1,09(0,95-1,25)$ & \\
\hline $1501-2924$ & $1,06(0,92-1,23)$ & & $0,98(0,84-1,13)$ & \\
\hline$<2925$ & 1,0 & & 1,0 & \\
\hline Situação conjugal & & $<0,001$ & & $<0,001$ \\
\hline Casada & 1,0 & & 1,0 & \\
\hline União consensual & $1,31(1,15-1,49)$ & & $1,30(1,14-1,48)$ & \\
\hline Solteira & $1,37(1,20-1,56)$ & & $1,41(1,24-1,61)$ & \\
\hline Divorciada ou separada & $1,76(1,43-2,17)$ & & $1,70(1,37-2,10)$ & \\
\hline Católica & & 0,277 & & 0,265 \\
\hline Sim & $0,94(0,84-1,05)$ & & $0,93(0,82-1,05)$ & \\
\hline Não & 1,0 & & 1,0 & \\
\hline Evangélica & & 0,110 & & 0,041 \\
\hline Sim & $1,09(0,98-1,21)$ & & $1,12(1,01-1,24)$ & \\
\hline Não & 1,0 & & 1,0 & \\
\hline \multicolumn{5}{|l|}{ Experiências familiar e de vida } \\
\hline Mãe já apanhou de algum parceiro ${ }^{b}$ & & $<0,001$ & & 0,001 \\
\hline $\operatorname{Sim}$ & $1,29(1,16-1,44)$ & & $1,21(1,09-1,36)$ & \\
\hline Não & 1,0 & & 1,0 & \\
\hline Violência sexual na infância & & $<0,001$ & & 0,004 \\
\hline $\operatorname{Sim}$ & $1,29(1,14-1,47)$ & & $1,23(1,07-1,41)$ & \\
\hline Não & 1,0 & & 1,0 & \\
\hline \multicolumn{5}{|l|}{ Características comportamentais } \\
\hline Dose de bebida alcoólica & & 0,013 & & 0,114 \\
\hline Nenhuma & 1,0 & & 1,0 & \\
\hline$<=2$ & $0,84(0,70-1,01)$ & & $0,85(0,69-1,04)$ & \\
\hline $2,1-8,0$ & $1,10(0,95-1,27)$ & & $1,10(0,95-1,28)$ & \\
\hline$>8,0$ & $1,21(1,01-1,44)$ & & $0,95(0,79-1,14)$ & \\
\hline Fumante & & $<0,001$ & & 0,005 \\
\hline Sim & $1,40(1,24-1,57)$ & & $1,20(1,06-1,36)$ & \\
\hline Não & 1,0 & & 1,0 & \\
\hline Histórico de uso drogas & & $<0,001$ & & $<0,001$ \\
\hline $\operatorname{Sim}$ & $1,58(1,43-1,74)$ & & $1,37(1,21-1,55)$ & \\
\hline Não & 1,0 & & 1,0 & \\
\hline
\end{tabular}


Tabela 3. Análise bruta e ajustada dos efeitos das variáveis socioeconômica, comportamentais, experiência familiar e de vida sobre a violência física perpetrada pelo parceiro íntimo ao longo da vida. Vitória, Espírito Santo, Brasil. Março a setembro, 2014.

\begin{tabular}{|c|c|c|c|c|}
\hline \multirow[b]{2}{*}{ Características sociodemográficas } & \multicolumn{2}{|c|}{ Análise bruta } & \multicolumn{2}{|c|}{ Análise ajustada } \\
\hline & $\begin{array}{l}\text { RP bruta } \\
\text { (IC95\%) }\end{array}$ & p-valor & $\begin{array}{c}\text { RP ajustada } \\
\text { (IC95\%) }\end{array}$ & p-valor \\
\hline Idade (anos) & & 0,613 & & 0,416 \\
\hline 20-29 & 1,0 & & 1,0 & \\
\hline $30-39$ & $0,91(0,75-1,12)$ & & $0,95(0,78-1,15)$ & \\
\hline $40-49$ & $1,06(0,86-1,30)$ & & $1,07(0,87-1,32)$ & \\
\hline $50-59$ & $0,97(0,77-1,23)$ & & $1,14(0,89-1,45)$ & \\
\hline Cor $^{\text {a }}$ & & 0,047 & & 0,430 \\
\hline Branca & 1,0 & & 1,0 & \\
\hline Parda & $1,35(1,06-1,71)$ & & $1,03(0,82-1,28)$ & \\
\hline Preta & $1,25(1,00-1,56)$ & & $1,14(0,89-1,45)$ & \\
\hline Escolaridade (em anos de estudo) & & $<0,001$ & & $<0,001$ \\
\hline $0-8$ & $1,62(1,35-1,95)$ & & $1,34(1,15-1,56)$ & \\
\hline 9 ou mais & 1,0 & & 1,0 & \\
\hline Renda familiar (em reais) & & $<0,001$ & & 0,005 \\
\hline$>=1500$ & $1,82(1,48-2,25)$ & & $1,41(1,13-1,76)$ & \\
\hline $1501-2924$ & $1,58(1,27-1,97)$ & & $1,38(1,10-1,72)$ & \\
\hline$<2925$ & 1,0 & & 1,0 & \\
\hline Situação conjugal & & $<0,001$ & & $<0,001$ \\
\hline Casada & 1,0 & & 1,0 & \\
\hline União consensual & $1,73(1,43-2,10)$ & & $1,61(1,32-1,97)$ & \\
\hline Solteira & $1,81(1,48-2,21)$ & & $1,83(1,50-2,24)$ & \\
\hline Divorciada ou separada & $2,58(1,86-3,57)$ & & $2,27(1,64-3,15)$ & \\
\hline Católica & & 0,847 & & 0,802 \\
\hline Sim & $0,98(0,84-1,15)$ & & $1,02(0,86-1,22)$ & \\
\hline Não & 1,0 & & 1,0 & \\
\hline Evangélica & & 0,031 & & 0,022 \\
\hline Sim & $1,19(1,01-1,39)$ & & $1,20(1,03-1,40)$ & \\
\hline Não & 1,0 & & 1,0 & \\
\hline \multicolumn{5}{|l|}{ Experiências familiar e de vida } \\
\hline Mãe já apanhou de algum parceiro ${ }^{b}$ & & $<0,001$ & & 0,003 \\
\hline Sim & $1,47(1,25-1,73)$ & & $1,27(1,08-1,49)$ & \\
\hline Não & 1,0 & & 1,0 & \\
\hline Violência sexual na infância & & $<0,001$ & & $<0,001$ \\
\hline Sim & $1,55(1,30-1,85)$ & & $1,45(1,18-1,77)$ & \\
\hline Não & 1,0 & & 1,0 & \\
\hline \multicolumn{5}{|l|}{ Características comportamentais } \\
\hline Dose de bebida alcoólica & & $<0,001$ & & 0,463 \\
\hline Nenhuma & 1,0 & & 1,0 & \\
\hline$<=2$ & $0,95(0,75-1,22)$ & & $1,03(0,79-1,35)$ & \\
\hline $2,1-8,0$ & $1,13(0,91-1,40)$ & & $1,19(0,96-1,47)$ & \\
\hline$>8,0$ & $1,65(1,33-2,05)$ & & $1,05(0,81-1,36)$ & \\
\hline Fumante & & $<0,001$ & & 0,002 \\
\hline Sim & $1,81(1,53-2,13)$ & & $1,34(1,11-1,62)$ & \\
\hline Não & 1,0 & & 1,0 & \\
\hline Histórico de uso drogas & & $<0,001$ & & $<0,001$ \\
\hline Sim & $2,09(1,81-2,42)$ & & $1,64(1,35-1,99)$ & \\
\hline Não & 1,0 & & 1,0 & \\
\hline
\end{tabular}

${ }^{\mathrm{a}} \mathrm{n}=957 ;{ }^{\mathrm{b}} \mathrm{n}=898$. 
Tabela 4 Análise bruta e ajustada dos efeitos das variáveis socioeconômica, comportamentais, experiência familiar e de vida sobre a violência sexual perpetrada pelo parceiro íntimo ao longo da vida. Vitória, Espírito Santo, Brasil. Março a setembro, 2014.

\begin{tabular}{|c|c|c|c|c|}
\hline \multirow[b]{2}{*}{ Características sociodemográficas } & \multicolumn{2}{|c|}{ Análise bruta } & \multicolumn{2}{|c|}{ Análise ajustada } \\
\hline & $\begin{array}{l}\text { RP bruta } \\
\text { (IC95\%) }\end{array}$ & p-valor & $\begin{array}{c}\text { RP ajustada } \\
\text { (IC95\%) }\end{array}$ & p-valor \\
\hline Idade (anos) & & 0,055 & & 0,111 \\
\hline 20-29 & 1,0 & & 1,0 & \\
\hline $30-39$ & $0,97(0,66-1,42)$ & & $0,97(0,66-1,42)$ & \\
\hline $40-49$ & $1,47(1,02-2,11)$ & & $1,37(0,94-2,01)$ & \\
\hline 50-59 & $1,37(0,92-2,03)$ & & $1,43(0,94-2,17)$ & \\
\hline Cor $^{\text {a }}$ & & 0,910 & & 0,485 \\
\hline Branca & 1,0 & & 1,0 & \\
\hline Parda & $0,97(0,65-1,43)$ & & $0,89(0,60-1,33)$ & \\
\hline Preta & $0,93(0,66-1,30)$ & & $0,81(0,58-1,14)$ & \\
\hline Escolaridade (em anos de estudo) & & $<0,001$ & & 0,001 \\
\hline $0-8$ & $1,53(1,22-1,83)$ & & $1,63(1,24-2,14)$ & \\
\hline 9 ou mais & 1,0 & & 1,0 & \\
\hline Renda familiar (tercis) & & 0,039 & & 0,106 \\
\hline$>=1500$ & $1,56(1,11-2,19)$ & & $1,30(0,92-1,86)$ & \\
\hline $1501-2924$ & $1,32(0,93-1,87)$ & & $0,97(0,67-1,41)$ & \\
\hline$<2925$ & 1,0 & & 1,0 & \\
\hline Situação conjugal & & 0,021 & & 0,015 \\
\hline Casada & 1,0 & & 1,0 & \\
\hline União consensual & $1,32(0,96-1,83)$ & & $1,31(0,94-1,82)$ & \\
\hline Solteira & $1,44(1,03-2,01)$ & & $1,55(0,38-1,68)$ & \\
\hline Divorciada ou separada & $2,40(1,26-4,54)$ & & $2,18(1,17-4,08)$ & \\
\hline Católica & & 0,586 & & 0,717 \\
\hline Sim & $0,93(0,71-1,22)$ & & $0,94(0,67-1,31)$ & \\
\hline Não & 1,0 & & 1,0 & \\
\hline Evangélica & & 0,035 & & 0,042 \\
\hline Sim & $1,33(1,02-1,74)$ & & $1,33(1,01-1,74)$ & \\
\hline Não & 1,0 & & 1,0 & \\
\hline \multicolumn{5}{|l|}{ Experiências familiar e de vida } \\
\hline Mãe já apanhou de algum parceiro ${ }^{b}$ & & 0,003 & & 0,028 \\
\hline Sim & $1,54(1,16-2,05)$ & & $1,37(1,03-1,84)$ & \\
\hline Não & 1,0 & & 1,0 & \\
\hline Violência sexual na infância & & $<0,001$ & & $<0,001$ \\
\hline Sim & $2,43(1,83-3,21)$ & & $2,08(1,50-2,88)$ & \\
\hline Não & 1,0 & & 1,0 & \\
\hline \multicolumn{5}{|l|}{ Características comportamentais } \\
\hline Dose de bebida alcoólica & & 0,070 & & 0,081 \\
\hline Nenhuma & 1,0 & & 1,0 & \\
\hline$<=2$ & $0,56(0,34-0,91)$ & & $0,91(0,41-1,15)$ & \\
\hline $2,1-8,0$ & $0,73(0,47-1,11)$ & & $0,70(0,45-1,08)$ & \\
\hline$>8,0$ & $0,83(0,48-1,46)$ & & $0,59(0,33-1,04)$ & \\
\hline Fumante & & 0,043 & & 0,292 \\
\hline Sim & $1,45(1,01-2,07)$ & & $1,24(0,83-1,89)$ & \\
\hline Não & 1,0 & & 1,0 & \\
\hline Histórico de uso de drogas & & $<0,001$ & & $<0,001$ \\
\hline Sim & $2,19(1,62-2,96)$ & & $2,06(1,46-2,91)$ & \\
\hline Não & 1,0 & & 1,0 & \\
\hline
\end{tabular}

${ }^{\mathrm{a}} \mathrm{n}=957 ;^{\mathrm{b}} \mathrm{n}=898$. 
42,3), com prevalência semelhante a outro estudo nacional ${ }^{15}$, e ainda que a frequência de violência sexual praticada pelo parceiro íntimo na vida tenha sido a mais baixa entre as violências pesquisadas ( $\mathrm{P}=18,0 \%$; IC95\% 15,7-20,5), esta se apresentou acima das prevalência revelada por outro estudo realizado no Brasil 13,6\% ${ }^{13}$. Acredita-se que vários fatores contribuam para que a violência sexual dentro de relações de parcerias estáveis seja de difícil reconhecimento e delimitação. Entre eles, o fato da mulher não entender o sexo forçado como violência, caso ela esteja casada ou viva com o agressor. Além disso, em alguns países, apesar de já se ter reconhecido o estupro, no matrimônio, como crime; em outros, o esposo ainda tem o direito legal de acesso sexual ilimitado à esposa ${ }^{16}$.

O estudo identificou a associação das violências com as variáveis socioeconômicas. O menor nível de escolaridade esteve associado à maiores prevalências de violência, corroborando outro estudo ${ }^{17}$. Acredita-se que o esclarecimento da mulher leva a menor tolerância à violência. Quanto mais a mulher se qualifica, mais chance tem de encontrar trabalho remunerado, melhorando assim a autoestima e a independencia ${ }^{18}$. Sugere-se ainda que mulheres com níveis educacionais mais altos teriam mais recursos para alcançar maior autonomia e poderiam ter mais habilidades para reconhecer e romper com relacionamentos abusivos ${ }^{15}$.

Evidenciou-se ainda, a associação da violência física ao longo da vida com os níveis de renda mais baixos. Nesse contexto, apesar da violência atingir a todos os grupos socioeconômicos, mulheres mais pobres são afetadas em maior proporção ${ }^{19}$. Esses achados provavelmente se explicam, pois a mulher em situação de violência, que apresenta baixas condições sociais e econômicas pode ter maiores dificuldades na tomada de decisão no sentido de romper com o relacionamento violento, considerando o fato de não apresentar autonomia financeira ${ }^{20}$.

Ainda, é importante refletir, que a exposição à violência possui efeitos negativos no desempenho escolar ${ }^{21}$. Esse agravo pode ter relação com a menor escolaridade das vítimas, e, por consequência com a renda mais baixa e ocupação de cargos menos valorizados ${ }^{22}$. Além disso, a maior prevalência de violência doméstica entre famílias mais pobres pode sugerir que o mecanismo de transmissão intergeracional de violência, pode estar relacionado à baixa mobilidade intergeracional de renda. Nota-se, uma relação negativa entre prevalência de violência doméstica e renda tanto em relação à renda dos pais quanto em relação à renda atual da mulher ${ }^{23}$.

Em consonância com a literatura ${ }^{15}$, o estudo também revelou alta prevalência de violência em mulheres separadas e divorciadas, sugerindo que muitas mulheres conseguem se libertar da situação de violência, rompendo o estereótipo de que a violência por parceiro íntimo é uma situação irremediavelmente crônica.

A condição referida "mãe vítima de violência por parceiro íntimo”, demonstrou associação com a violência. A literatura aponta que um ambiente familiar com histórico de violência presenciada ou sofrida na infância, pode desencadear um espaço para o comportamento violento. Além disso, pode sugerir uma vulnerabilidade do gênero, altamente relacionada com a violência sofrida na vida adulta. Dessa maneira, sugere-se que padrões de violência na família podem se reproduzir através de gerações ${ }^{24}$.

As mulheres fumantes e aquelas com histórico de uso de drogas apresentaram maiores prevalências de violências física e psicológica ao longo da vida. Já a violência sexual revelou associação somente com o uso de drogas. A literatura confirma entre as mulheres com comportamento de alto risco, como uso de drogas, apresentam maiores frequências de violência praticada pelo parceiro íntimo ${ }^{13}$. Além disso, vale refletir que vivenciar uma situação de violência pode tornar a mulher mais propensa a relatar o uso de álcool e o consumo de drogas ilícitas, a fim de lidar com o evento violento ${ }^{25,26}$, ou ainda, a violência, em suas várias formas, podem levar ao ato de fumar de modo a minimizar e suportar esse agravo ${ }^{27}$.

A presente pesquisa revela que as violências psicológica, física e sexual apresentaram associação significativa com a religião evangélica, achado que se assemelha ao pontuado em recente revisão sistemática que mostra que a violência doméstica está associada à religião ${ }^{28}$. Nesse sentido, a literatura sugere a busca pela religião como uma forma de suportar ou superar a violência vivida $^{29}$.

Ainda, vale refletir que o enfrentamento da violência, ou seja, o ato de denunciar ou decidir romper com o silêncio diante da situação de violência apresenta aspectos facilitadores e dificultadores. Atitudes pessoais, como sentimentos de exaustão, revolta e consciência do potencial risco de vida estão entre os aspectos facilitadores, já os fatores dificultadores, incluem o medo, a culpa e a vergonha, bem como, entraves de âmbito familiar, material e institucional ${ }^{30}$. Nesse contexto, é necessário a alocação adequada de recursos hu- 
manos para a expansão dos centros de atenção às vítimas de violência, considerando a violência contra a mulher um importante problema de saúde pública ${ }^{31}$.

Algumas limitações precisam ser destacadas no estudo. A primeira delas é o fato de ter sido realizado em serviço de saúde. Dessa forma, pode ter sido menor a participação de mulheres de melhor condição socioeconômica, com acesso aos planos de saúde. E, embora se saiba que a ocorrência de violência foi menor entre essas mulheres é possível que as associações fossem até mesmo mais fortes com a condição socioeconômica mais baixa. Além desta, pode ter sido menor a participação de mulheres em situação de violência que poderiam sentirem-se inibidas de frequentarem a US. Todavia, as elevadas prevalências de violências evidenciadas podem sugerir que esse problema seria ainda maior, caso essas mulheres tivessem sido incluídas no estudo. Finalmente, o delineamento transversal impede assegurar a relação de causa e efeito entre as variáveis. Entretanto, as fortes associações demonstram a importância de estabelecer medidas de intervenções em relação às exposições que desencadeiam maior ocorrência de vitimização.

\section{Colaboradores}

IB Santos participou da interpretação dos dados, redação do artigo, revisão crítica e aprovação da versão a ser publicada. MHC Amorim participou da revisão crítica e aprovação da versão a ser publicada. DP Gigante participou do delineamento, análise, interpretação dos dados, revisão crítica e aprovação da versão a ser publicada. PMA Maciel participou da revisão crítica e aprovação da versão a ser publicada. FMC Leite participou da concepção, delineamento, análise, interpretação dos dados e aprovação da versão a ser publicada.

\section{Conclusão}

O presente estudo atendeu ao objetivo proposto e evidenciou as elevadas prevalências de violência por parceiro íntimo, praticadas ao longo da vida, entre as usuárias da atenção primária do município de Vitória, e a associação das violências psicológica, física e sexual com as condições sociodemográficas, comportamentais e experiências pessoal e materna de violência.

Por fim, compreende-se que esta pesquisa traz importantes contribuições à área da saúde pública, indicando novos olhares e caminhos para o cuidado às mulheres em situação de violência. Diante da magnitude do problema revelado, confirma-se a necessidade da implantação de instrumentos sensíveis à detecção e à abordagem da violência no cotidiano dos serviços de atenção primária, promovendo, assim, maior visibilidade à violência contra a mulher, bem como a promoção de um cuidado qualificado, que atenda essas vítimas de forma integralizada.

\section{Financiamento}

Fundação de Amparo à Pesquisa e Inovação do Espírito Santo (FAPES). 


\section{Referências}

1. United Nations (UN). Declaration on the elimination of violence against women. General Assembly. Nova York: UN; 1993.

2. Bandeira LM. Violência de gênero: a construção de um campo teórico e de investigação. Rev Soc Estado 2014; 29(2):449-469.

3. World Health Organization (WHO). Global and regional estimates of violence against women: prevalence and health effects of intimate partner violence and non-partner sexual violence. Genebra: WHO; 2013.

4. Schraiber LB, D’Oliveira AFPL, França-Junior I, Diniz S, Portella AP, Ludermir AB, Valença O, Couto MT. Prevalence of intimate partner violence against women in regions of Brazil. Rev Saude Publica 2007; 41(5):797-807.

5. Lucena KDT, Vianna RPT, Nascimento JA , Campos HFC, Oliveira ECT. Association between domestic violence and women's quality of life. Rev Latino Am Enferm 2017; 25:e2901.

6. World Health Organization (WHO), London School of Hygiene and Tropical Medicine. Preventing intimate partner and sexual violence against women: taking action and generating evidence. Genebra: WHO; 2010.

7. Oliveira EN, Jorge MSB. Violência contra a mulher: sofrimento psíquico e adoecimento mental. RENE 2007; 8(2):93-100.

8. Garbin CAS, Dias IA, Rovida TAS, Garbin AJI. Desafios do profissional de saúde na notificação da violência: obrigatoriedade, efetivação e encaminhamento. Cien Saude Colet 2015; 20(6):1879-1890.

9. Moreira TNF, Martins CL, Feuerwerker LCM, Schraiber LB. A construção do cuidado: o atendimento às situações de violência doméstica por equipes de Saúde da Família. Saude Soc 2014; 23(3):814-827.

10. Instituto Brasileiro de Geografia e Estatística (IBGE). População no último censo. Espírito Santo [página na Internet]. 2010 [acessado 2018 Abr 15] Disponível em: https://cidades.ibge.gov.br/brasil/es/vitoria/panorama

11. Atlas do Desenvolvimento Humano no Brasil. O Atlas: Consulta [página na Internet]. 2010 [acessado 2018 Abr 15] Disponível em: http://www.atlasbrasil. org.br/2013/pt/consulta/

12. Schraiber LB, Latorre MRDO, França Jr I, Segri NJ, D'Oliveira AFPL. Validity of the WHO VAW study instrument for estimating gender-based violence against women. Rev Saude Publica 2010; 44(4):658666.

13. Barros EM, Falbo Neto GH, Lucena SG, Ponzo L, Pimentel AP. Prevalência e fatores associados à violência por parceiro íntimo em mulheres de uma comunidade em Recife/Pernambuco, Brasil. Cien Saude Colet 2016; 21(2):591-598.

14. Moreira KAP, Costa AM, Marques JF, Fernandes AFC, Henriques ACPT. Violência contra a mulher: caracterização de casos atendidos em um centro estadual de referência. RENE 2011; 12(3):627-635.
15. Vieira EM, Perdona GSC, Santos MA. Fatores associados à violência física por parceiro íntimo em usuárias de serviços de saúde. Rev Saude Publica 2011; 45(4):730-737.

16. Scaranto CAA, Biazevic MGH, Michel-Crosato E. Percepção dos agentes comunitários de saúde sobre a violência contra a mulher. Psicol Cien Prof 2007; 27(4):694-705.

17. Leite FMC, Amorim MHC, Wehrmeister FC, Gigante DP. Violence against women, Espírito Santo, Brazil. Rev Saude Publica 2017; 51:33.

18. Adeodato VG, Carvalho RR, Siqueira VR, Soua FGM. Qualidade de vida e depressão em mulheres vítimas de seus parceiros. Rev Saude Publica 2005; 39(1):108113.

19. Jewkes R, Sen P, Garcia-Moreno C. Sexual violence. In: Krug EG, Dahlberg LL, Mercy JA, Zwi AB, Lozano R, editores. World report on violence and health. Genebra: WHO; 2002. p. 147-174.

20. Fortuna SLA. As estratégias de enfrentamento da violência doméstica: um estudo sobre Guarapuava. ex cequo 2011; 24:139-151.

21. Carneiro JB, Gomes NP, Estrela FM, Santana JD, Mota RS, Erdmann AL. Violência conjugal: repercussões para mulheres e filhas(os). Esc Anna Nery 2017; 21(4):e20160346.

22. Martins JC. Determinantes da violência doméstica contra a mulher no Brasil [dissertação]. Viçosa: Universidade Federal de Viçosa; 2017.

23. Carvalho JR, Oliveira VH. Pesquisa de Condições Socioeconômicas e Violência Doméstica e Familiar contra a Mulher PCSVDF [relatório na Internet]. Fortaleza; 2017 [acessado 2018 Abr 15]. Disponível em: http:// www.onumulheres.org.br/wp-content/uploads/ 2017/11/violencia_domestica_geracoes_out_17.pdf

24. Zancan N, Wassermann V, Lima GQ. A violência doméstica a partir do discurso de mulheres agredidas. Pensando Fam 2013; 17(1):63-76.

25. Sapori LF, Sena LL. Crack e violência urbana. In: Ribeiro M, Laranjeira R, organizadores. O tratamento do usuário de crack. Porto Alegre: Editora Artmed; 2012. p. 74-91.

26. El-Bassel N, Gilbert L, Wu E, Go H, Hill J. Relationship between drug abuse and intimate partner violence: a longitudinal study among women receiving methadone. Am J Public Health 2005; 95(3):465-470.

27. Borges MTT, Simões-Barbosa RH. Cigarro "companheiro": o tabagismo feminino em uma abordagem crítica de gênero. Cad Saude Publica 2008; 24(12):2834-2842.

28. Agumasie S, Bezatu M. Domestic violence against women and associated factors in Ethiopia; systematic review. Reprod Health. 2015; 12:78.

29. St. Vil NM, Sabri B, Nwokolo V, Alexander KA, Campbell JC. A Qualitative Study of Survival Strategies Used by Low-Income Black Women Who Experience Intimate Partner Violence. Soc Work 2017; 62(1):6371. 
30. Leite FMC, Moura MAV, Penna LHG. Percepções das mulheres sobre a violência contra a mulher: uma revisão integrativa da literatura. Av Enferm 2013; 31(2):136-143.

31. Bernardino IM, Barbosa KGN, Nóbrega LM, Cavalcante GMS, Ferreira EF, d'Avila S. Violência contra mulheres em diferentes estágios do ciclo de vida no Brasil: um estudo exploratório. Rev Bras Epidemiol 2016; 19(4):740-752.

Artigo apresentado em 27/12/2017

Aprovado em 11/09/2018

Versão final apresentada em 13/09/2018 